\title{
A LOWER ESTIMATE FOR EXPONENTIAL SUMS
}

\author{
BY C. A. BERENSTEIN ${ }^{1}$ AND M. A. DOSTAL
}

Communicated by François Treves, October 8, 1973

1. Introduction. In this note we present two theorems on exponential sums (see Theorems 1 and 2 below). Although seemingly unrelated, both results are motivated by the study of a certain type of lower estimates of exponential sums in the complex domain. Thus while Theorem 2 is related to the validity of this estimate for all discrete exponential sums ${ }^{2}$, Theorem 1 essentially says that even a milder estimate of this kind does not hold for a whole class of continuous exponential sums (i.e. for certain Fourier transforms).

In addition to the usual notation of the theory of distributions (cf. [2], [3], [7]), the following symbols will be used throughout this note. Given a distribution $\Phi \in \mathscr{E}^{\prime}=\mathscr{E}^{\prime}\left(\boldsymbol{R}^{n}\right)$, the symbol $[\Phi](\{\Phi\}$ resp.) denotes the convex hull of the support of $\Phi$ (singular support of $\Phi$, resp.). For $A \subset \boldsymbol{R}^{n}, h_{A}$ is the supporting function of $A$, i.e. $h_{A}(\lambda)=\sup _{x \in A}\langle x, \lambda\rangle$, $\lambda \in \boldsymbol{R}^{n}$. For $\zeta \in C^{n}$ and $r>0, \Delta=\Delta(\zeta ; r)$ is the closed polydisk with center $\zeta$ and radius $r$; and, if $g\left(\zeta^{\prime}\right)$ is any continuous function on $\Delta(\zeta ; r)$, we shall write

$$
|g(\zeta)|_{r}=\max _{\zeta^{\prime} \in \Delta}\left|g\left(\zeta^{\prime}\right)\right|
$$

2. Indicators of smooth convex bodies.

DEFINITION. Let $\Phi \in \mathscr{E}^{\prime}$ be such that

$$
\{\Phi * \Psi\}=\{\Phi\}+\{\Psi\} \quad\left(\forall \Psi \in \mathscr{E}^{\prime}\right)
$$

Then $\Phi$ will be called a good convolutor.

The relationship of being a good convolutor to the solvability of the convolution equation $\Phi * u=f$ in the appropriate distribution spaces was discovered by L. Hörmander [7], and since then it was discussed by several authors (for references, cf. [2, Chapter I]). However, it is usually not easy to decide whether a given distribution $\Phi$ is a good convolutor or not.

AMS (MOS) subject classifications (1970). Primary 33A10, 32A15, 47G05.

Key words and phrases. Exponential-polynomials, Fourier transforms, several complex variables, convolution equations.

${ }^{1}$ The first author was supported in part by the U.S. Army Research Office (Durham)

${ }^{2}$ And more generally, for all exponential polynomials. 
Moreover, few good convolutors are known, and as Theorem 1 below will indicate, even distributions of a very simple nature may fail to be good convolutors.

It can be shown [4, Proposition 2] that the following condition on $\hat{\Phi}$ is sufficient for $\Phi$ to be a good convolutor:

Condition $\left(\mathrm{R}_{\omega}\right)$. There exist constants $t \geqq 0, r>0, c>0$ and $A$ real (all depending on $\Phi$ ) so that (cf. (1))

$$
|\Phi(\zeta)|_{r} \geqq c(1+|\xi|)^{A} \exp \left(h_{[\Phi]}(\eta)\right)
$$

for all $\zeta=\xi+i \eta \in C^{n}$ such that $|\xi| \geqq t$ and $|\eta|>t \log (1+|\xi|)$.

Since any distribution $\Phi$ with finite support satisfies condition $\left(R_{\omega}\right)$ (cf. [4, Proposition 6]), we thus obtain a result of Hörmander [7], [8], according to which all distributions with finite support are good convolutors. This in turn can be used to prove the following statement (cf. [4, Proposition 6]):

Let $P$ be an arbitrary compact convex polyhedron in $\boldsymbol{R}^{n}$ and $\chi_{P}$ the distribution defined by the characteristic function of $P$. Then $\chi_{P}$ satisfies condition $\left(\mathrm{R}_{\omega}\right)$, hence $\chi_{P}$ is a good convolutor. The same conclusion holds for the surface measure $\chi_{\partial P}$ of density 1 , i.e.

$$
\chi_{\hat{\partial} P}(\phi)=\int_{\partial P} \phi(x) d s_{x}, \quad(\phi \in \mathscr{E})
$$

where $d s_{x}$ is the surface element.

It seems natural to ask whether this proposition holds for smooth convex bodies $P$ as well. At the first glance it seems that it does. Indeed, if, for instance, $P$ is any ellipsoid in $R^{n}$, then the distribution $\Phi=\chi_{P}$ satisfies the following weaker version of (2) (cf. the concluding remark in [5]):

$$
\{\Psi\} \subseteq\{\Phi * \Psi\}-\{\Phi\} \quad\left(\forall \Psi \in \mathscr{E}^{\prime}\right) .
$$

Therefore, it is rather surprising that this particular $\Phi$ is not a good convolutor [5, Proposition 4]. The following theorem sheds more light on this peculiar situation.

THEOREM 1. Let $P$ be a convex body in $\boldsymbol{R}^{n}(n>1)$ with a $C^{\infty}$-boundary $\partial P$. Moreover, it is assumed that the Gaussian curvature of $\partial P$ never vanishes, i.e. $K(x)>0$ for every $x \in \partial P$. Then neither $\chi_{P}$ nor $\chi_{\partial P}$ is a good convolutor.

REMARK. Both assumptions on $\partial P$ (i.e. smoothness and $K>0$ ) can be substantially relaxed.

The proof of Theorem 1 is based on a detailed study of the asymptotic behavior of the functions $\hat{\chi}_{P}$ and $\hat{\chi}_{\partial P}$ in the complex domain. For $\zeta$ real, estimates of this kind were previously derived by numerous authors (cf. [9], [10], [11] and the references given in [10], [11]). However, for our 
purposes these estimates must be sharpened. As an illustration, consider the case of the convex surface $S=\partial P$. Given $\zeta=\xi+i \eta \in C^{n}$ with $\xi \neq 0$, write $r=|\xi|$ and consider $\zeta=r \omega+i \eta$ with $\omega$ fixed. Let $x^{j}=\left(x_{1}^{j}, \cdots, x_{n}^{j}\right) \in S$ $(j=0,1)$ be the points

$$
x_{v}^{j}=\partial h_{S}\left((-1)^{j} \xi\right) / \partial \xi_{v} \quad(v=1, \cdots, n) .
$$

Fix arbitrarily the open subsets $S^{k}(k=0,1,2)$ of $S$ so that $S=\bigcup_{k} S^{k}$, $S^{0} \cap S^{1}=\varnothing, x^{j} \in S^{j} \mid S^{2}(j=0,1)$. Then for any $q>n / 2$ and $\nu>0$ there exist positive numbers $a_{j}, b_{j}$ and $c_{v}$ such that

$$
\begin{aligned}
\hat{\chi}_{S}(\zeta)= & (1-i)^{n-1}\left(\frac{\pi}{2}\right)^{n-1} r^{(1-n) / 2} \sum K\left(x^{j}\right)^{-1 / 2} \exp \left(-i\left\langle x^{j}, \zeta\right\rangle\right) \\
& +I_{1}+I_{2}+I_{3} ; \\
\left|I_{1}(\zeta)\right| \leqq & r^{-n / 2}(1+|\eta|)^{q} \sum a_{j} \exp \left(\left\langle x^{j}, \eta\right\rangle\right), \\
\left|I_{2}(\zeta)\right| \leqq & r^{-q}(1+|\eta|)^{2 q} \sum b_{j} \exp \left[h_{S^{j}}(\eta)\right], \\
\left|I_{3}(\zeta)\right| \leqq & c_{v} r^{-v}(1+|\eta|) \exp \left[h_{S^{2}}(\eta)\right],
\end{aligned}
$$

where $\sum=\sum_{j=0,1}$. Formula (4) combined with a result of Hörmander [8] yields Theorem 1 for $\chi_{\partial P}$. Asymptotic expansions similar to (4) hold for $\hat{\chi}_{P}$ as well as for the Fourier transforms of certain measures with nonconstant density.

3. The discrete case. Generalization of Ritt's theorem. In this part we shall consider finite exponential sums, and more generally, exponential polynomials in several complex variables. If $H$ is an exponential polynomial, i.e. a function of the form

$$
H(\zeta)=\sum_{j=1}^{s} h_{j}(\zeta) \exp \left(\left\langle\theta_{j}, \zeta\right\rangle\right) \quad\left(\zeta \in C^{n}\right)
$$

with complex frequencies $\theta_{j} \in C^{n}$ and polynomial coefficients $h_{j}$, the greatest common divisor of the $h_{j}$ 's, $d_{H}=\left(h_{1}, \cdots, h_{s}\right)$, will be called the content of $H$. Moreover, we shall write $\mathfrak{C}_{H}(\zeta)=\max _{j} \operatorname{Re}\left\langle\theta_{j}, \zeta\right\rangle$. Henceforth an exponential sum will mean a function of the form (5) with all coefficients $h_{j}$ constant. The following lower estimate of exponential polynomials was proved in [3], [5]:

$\left(\mathrm{R}_{0}\right)$ Given an exponential polynomial $H$ and an arbitrary $\varepsilon>0$, there exists $C=C(\varepsilon, H)>0$ such that for every $\zeta \in C^{n}$ and any $f$ analytic in $\Delta(\zeta ; \varepsilon)$,

$$
|f(\zeta)| \exp \left(\mathfrak{C}_{H}(\zeta)\right) \leqq C|f(\zeta) H(\zeta)|_{\varepsilon} \cdot{ }^{3}
$$

\footnotetext{
${ }^{3}$ Obviously, estimate $\left(R_{0}\right)$ is much stronger than $\left(R_{\omega}\right)$.
} 
In this section we shall discuss the following

Question. Let $F$ and $G$ be exponential polynomials in $n$ variables such that the function $H=F / G$ is entire. What can be said about the structure of $H$ ? In particular, when is $H$ an exponential polynomial?

Simple examples show that $H$ need not be an exponential polynomial (e.g., $n=1, F=\sin \zeta, G=\zeta$ ). On the other hand, if $F$ and $G$ are exponential sums in one variable such that $H$ is entire, then, according to a theorem of Ritt [12], $H$ is also an exponential sum. Different proofs of Ritt's theorem were given by H. Selberg, P. D. Lax and A. Shields (cf. the references in [12], [13]). In particular, Shields [13] proves that $H$ is an exponential polynomial as long as it is entire and $G$ is an exponential sum. He also mentions that, according to an unpublished result of W. D. Bowsma, the last assumption may be replaced by $d_{G}=1$. Finally, Avanissian and Martineau [1] generalized the original Ritt's theorem to arbitrary $n>1$. The following theorem contains all these results as special cases. Moreover, it shows that the above counterexample is in a certain sense the best possible:

THEOREM 2. Let $F, G, H$ be as above ( $n \geqq 1$ arbitrary). Then there exists an exponential polynomial $E$ and a polynomial $Q$ such that $H=E / Q$. Hence we may assume $\left(d_{E}, Q\right)=1$. Then $E$ and $Q$ are determined uniquely ${ }^{4}$ and $Q$ divides $d_{G}$.

The starting point for the proof of Theorem 2 is the following assertion: Let $f, g, h$ be the analytic functionals whose Fourier-Borel transforms are $F, G, H$ respectively. Then $h$ is carried by the polyhedron defined by $\mathfrak{C}_{F}-$ $\mathfrak{C}_{G}$. This in turn follows from $\left(\mathrm{R}_{0}\right)$.

The proofs together with applications of the above theorems will appear elsewhere.

\section{BIBLIOGRAPHY}

1. V. Avanissian, Oral communication, 1970.

2. C. A. Berenstein and M. A. Dostal, Analytically uniform spaces and their applications to convolution equations, Lecture Notes in Math., vol. 256, Springer-Verlag, Berlin and New York, 1972.

3. - - Some remarks on convolution equations, Ann. Inst. Fourier 23 (1973), 55-74.

4. - On convolution equations. I, L'Analyse Harmonique dans le Domaine Complexe, Lecture Notes in Math., vol. 336, Springer-Verlag, Berlin and New York, 1973, pp. 79-94.

5. - On convolution equations. II, Proc. Colloq. Anal. Rio de Janeiro, 1972 (to appear).

6. M. A. Dostal, An analogue of a theorem of Vladimir Bernstein and its applications to singular supports of distributions, Proc. London Math. Soc. (3) 19 (1969), 553-576. MR 40 \#3302.

\footnotetext{
4 Up to a constant multiple.
} 
7. L. Hörmander, On the range of convolution operators, Ann. of Math. (2) 76 (1962), 148-170. MR 25 \#5379.

8. - Supports and singular supports of convolutions, Acta Math. 110 (1963), 279-302. MR 27 \#4070.

9. F. John, Bestimmung einer Funktion aus ihren Integralen über gewisse Mannigfaltigkeiten, Math. Ann. 109 (1934), 488-520.

10. W. Littman, Fourier transforms of surface-carried measures and differentiability of surface averages, Bull. Amer. Math. Soc. 69 (1963), 766-770. MR 27 \#5086.

11. - Decay at infinity of solutions to partial differential equations with constant coefficients, Trans. Amer. Math. Soc. 123 (1966), 449-459. MR 33 \#6110.

12. J. F. Ritt, On the zeros of exponential polynomials, Trans. Amer. Math. Soc. 31 (1929), 680-686.

13. A. Shields, On quotients of exponential polynomials, Comm. Pure Appl. Math. 16 (1963), 27-31. MR 26 \#6411.

Department of Mathematics, University of Maryland, College Park, MaryLAND 20742

Department of Mathematics, Stevens Institute of Technology, Hoboken, New JERSEY 07030

Instituto de Matematica Pura e Aplicada, Rio de Janeiro, Brazil 